\title{
THE RELATIONSHIP BETWEEN ACTUAL AND SELF- PERCEIVED PHYSICAL FITNESS IN ADOLESCENCE
}

\author{
Robert Bezjak ${ }^{1}$ and Saša Cecić Erpič ${ }^{2}$ \\ ${ }^{1}$ Independent Researcher \\ ${ }^{2}$ University of Ljubljana, Faculty of Sport, Slovenia
}

Original scientific paper

DOI: $10.26582 / \mathrm{k} .53 .1 .5$

\begin{abstract}
:
The purpose of this study was to assess the relationship between physical self-concept and actual indicators of physical fitness in early and mid-adolescence. Physical self-concept plays a significant role in adolescence, as this phase of life is characterised by many physical, mental, and emotional changes. A total of 427 Slovenian boys and girls from two age groups (early and middle adolescence) were studied. Physical self-concept was assessed with the Slovenian version of Physical Self-Description Questionnaire, while the Sports-educational Chart was used to measure physical fitness. The results showed that the older group scored better on all measures of physical fitness, except for general endurance. In terms of age-related differences in physical self-concept, older adolescents showed poorer physical perceptions of their strength and flexibility and higher global self-esteem compared to their younger peers. Correlation analysis showed that physical self-concept and actual indicators of physical fitness were related, despite incomplete overlap between psychological dimensions and objective measures. The obtained results can be transferred to physical education, as the development of a positive physical self-concept is one of the most important educational goals.
\end{abstract}

Key words: physical self-concept, actual physical fitness, adolescence, self-perceptions

\section{Introduction}

The perception of one's body and physical abilities plays an important role in the formation of self-concept. At any time in life, and especially in adolescence, experiencing one's body as something redundant can lead to a negative self-image, various unpleasant body-related feelings, social anxiety, and low self-esteem (Lintunen, 1999).

The concept of physical fitness can be divided into health-related fitness and skill-related fitness (Malina, Bouchard, \& Bar-Or, 2004). Health-related fitness includes the components of cardiorespiratory endurance, muscular endurance, muscular strength, body composition, and flexibility. Skillrelated fitness includes factors such as agility, balance, coordination, speed, strength, and reaction time (Jaakkola \& Washington, 2011). Methods for analysing physical fitness in children and adolescents typically include both fitness domains. In Slovenia, Strel and colleagues (Kovač, Jurak, Starc, Leskošek, \& Strel, 2011; Strel, et al., 1997, 2003; Starc, Strel, \& Kovač, 2010) have developed the Sports-educational Chart (SEC) that includes eight motor tasks and three anthropometric measures for measuring physical characteristics and motor skills in childhood and adolescence (for detailed information on SEC see Methods section). Numerous studies (for a review see Malina, et al., 2004) have shown that physical fitness in childhood and adolescence is an important factor influencing engagement in physical activity in adulthood. Engagement in physical activity in adulthood can be explained by several socio-cognitive models that hypothesise the involvement of several cognitive variables in this process. Physical self-perception is one of these cognitive attributes (Jaakkola \& Washington, 2011).

According to Marsh, Richards, Johnson, Roche, and Tremayne (1994), physical self-concept is considered to be a multidimensional subdomain of the general self-concept. It includes various characteristics such as health, strength, appearance, fitness, and physical activity (Marsh, 1993; Marsh, et. al., 1994). Although adopting a universally shared definition of physical self-concept seems difficult, it is reasonable to assert that it embraces all the knowledge, feelings, memories, and experiences that an individual associates with his or her body (Fox, 1998). During adolescence, an individual experiences many body-related changes, gender differences become apparent, and higher performance is achieved in most physical abilities. Therefore, 
physical self-concept is believed to play a crucial role during adolescence.

The relationship between physical self-perceptions and physical fitness has been demonstrated in many studies with samples of children (e.g., Hagger, Chatzisarantis, Biddle, \& Orbell, 2001; McIntyre, Parker, Chivers, \& Hands, 2018) and adolescents (e.g., Carcamo-Oyarzun, Estevan, \& Herrmann, 2020; Carraro, Scarpa, \& Ventura, 2010; Jaakkola \& Washington, 2011; Labbrozzi, Robazza, Bertollo, Bucci, \& Bortoli, 2013). According to Sonstroem's (1998) theory, physical fitness influences self-esteem through physical competence. Marsh (1993) developed a specific test of physical self-concept, the Physical Self-Description Questionnaire (PSDQ, for detailed information on the PSDQ see Methods section). It is one of the most widely used physical self-concept instruments that has been internally and externally validated.

Currently, there are no studies on the relationship between physical self-concept and physical fitness that include Slovenian adolescents. In particular, it is important to know how young and middle-aged adolescents perceive their physical self in relation to their fitness level and whether there are age-related differences in actual and self-perceived physical fitness. The present study focused on the relationship between physical self-concept and actual physical fitness in a sample of Slovenian early and middle adolescents. Therefore, this study had two aims: first, to analyse age-related differences in actual and self-perceived physical fitness, and second, to evaluate the relationship between the self-perceived physical fitness and its objective measures.

\section{Method}

\section{Participants}

Participants were a sample of $224\left(n_{\text {boys }}=101\right.$, $\mathrm{n}_{\text {girls }}=123$ ) primary school students and 203 ( $\mathrm{n}_{\text {boys }}$ $=83, \mathrm{n}_{\text {girls }}=117$ ) secondary school students from different randomly selected schools in Slovenia. Participants from the primary school were the $5^{\text {th }}$ and $7^{\text {th }}$ grade students (subjects' age varied between 12 and 14 years; $\mathrm{M}=13.34, \mathrm{SD}=0.75$ ) and participants from the secondary school were the $1^{\text {st }}$ and $3^{\text {rd }}$ grade students (their age varied between 16 and 18 years; $M=16.70, S D=0.67)$. The younger group represents the early adolescence stage (EA), while the older group represents the middle adolescence stage (MA).

\section{Instruments}

The Physical Self-Description Questionnaire (PSDQ; Marsh, et al., 1994) was used to assess physical self-concept. The PSDQ measures nine specific components of physical self (appearance, strength, fitness/endurance, flexibility, health, coordination, physical activity, sport competence, body fat) and two global components (Global Physical Self-Concept, Global Self-Esteem). The PSDQ consists of 70 items rated on a six-point Likert scale (1: strongly disagree, 6: strongly agree), with higher scores indicating higher physical self-concept. All negatively worded items (21 in total) are reversely scored and merged with other scores on the appropriate scale. Each scale consists of six items, except health and self-esteem (eight items each). The questionnaire was translated and adapted to the characteristics of the Slovenian sample by the authors of the present article. The Slovenian version of the PSDQ has good internal consistency (alphas range from .72 to .88) and validity (Bezjak, 2016).

Physical fitness was assessed using the Sportseducational Chart (SEC; Strel, et al., 1997). It measures eight components of physical fitness: general endurance [T600; 600-metre run test], static strength, i.e. muscular endurance of the shoulder girdle and arms [bent-arm hang test], sprint speed [60-metre run test], explosive strength [standing long jump test], coordination of the whole-body movements [obstacle course backward test], flexibility [forward bending on the bench test], speed of alternating movements [hand tapping test], and endurance strength, i.e., strength of abdominal muscles [sit-ups]. In addition to these motor measures, one morphological measure was collected [upper arm skinfold]. With the exception of the upper arm skinfold, the 60- and 600-metre run tests, higher scores represent better test results. All motor skill data were obtained from the Slovenian National Database of Motor Skills and Physical Characteristics (Strel, et al., 1997).

\section{Procedure}

A university human research ethics committee granted approval for this study. Parents or guardians provided written informed consents. All physical fitness assessments were conducted in schools during physical education classes as part of the National Physical Fitness Study. During the assessment, special care was taken to limit the possibility of comparing test scores between students. The PSDQ was administered to participants in a group class setting during the same week as the physical fitness assessments. Because students did not receive the physical fitness test results and the administration of the PSDQ did not occur on the exact same day, it can be assumed that the actual physical fitness test results did not influence the later ones.

Students answered to the PSDQ items voluntarily and anonymously, while the participation in the physical fitness tests (SEC) was mandatory, as they were part of the PE curriculum. 


\section{Data analysis}

Participants' scores on the PSDQ and SEC were summarized using descriptive statistics. The one-way ANOVA was used to evaluate differences in actual and self-perceived physical fitness between the students of different age groups. Since gender could affect both measures (physical tests and perceived self-concept), ANCOVA test was performed to avoid its effects in the comparison between the two age groups, using gender as a covariate.

The relationship between subjective and objective measures of physical self (i.e. between measures obtained with SEC - actual physical fitness and measures obtained with PSDQ - perceived physical fitness) was determined with bivariate Pearson correlations.

\section{Results}

\section{Differences by age}

Table 1 shows descriptive statistics of actual physical fitness measures by age. Results show that the primary and secondary school students differed significantly in all but two of the measures of actual physical fitness (see Table 1). The older students scored better on the hand tapping test, standing long jump, obstacle course backward test, forward bending on the bench test, as well as bent-arm hang test. The older group of students also achieved a higher number of sit-up repetitions and ran faster on the 60-metre course. However, there were no significant differences between the two groups in upper arm skinfold and 600-metre run scores.

Descriptive statistics and one-way ANOVA results for the PSDQ measures are presented in Table 2. Differences between the age groups were significant only for two specific components of physical self, i.e., strength and flexibility, and for global self-esteem. Mean scores indicated that the older students showed poorer physical perceptions of their strength and flexibility compared to the younger students. On the other hand, the results indicated that the older adolescents showed higher global self-esteem than their younger peers.

Since gender could have an influence on both sets of measures (physical tests and perceived self-concept), the statistical test ANCOVA was performed to avoid its influence. The results of one-way ANCOVA using gender as a covariate are presented in Tables 3 and 4. Table 3 shows the adjusted means and the ANCOVA used to test differences between the two age groups for the actual physical fitness variables, adjusted for

Table 1. Descriptive statistics and testing differences between age groups-actual physical fitness variables (assessed with Sportseducation Chart)

\begin{tabular}{|c|c|c|c|c|c|c|c|c|c|}
\hline Variable & $\begin{array}{l}\text { Age } \\
\text { group }\end{array}$ & $\mathrm{N}$ & M & SD & Min & Max & $\mathrm{df}$ & $\mathrm{F}$ & Sig. \\
\hline \multirow{2}{*}{ Upper-arm skinfold* } & EA & 180 & 12.13 & 5.54 & 3 & 31 & \multirow{2}{*}{1,303} & \multirow{2}{*}{1.10} & \multirow{2}{*}{.296} \\
\hline & MA & 126 & 12.86 & 6.54 & 3 & 41 & & & \\
\hline \multirow{2}{*}{ Hand tapping } & EA & 179 & 42.37 & 4.83 & 27 & 57 & \multirow{2}{*}{1,302} & \multirow{2}{*}{117.24} & \multirow{2}{*}{.000} \\
\hline & MA & 125 & 48.38 & 4.66 & 34 & 63 & & & \\
\hline \multirow{2}{*}{ Standing long jump } & EA & 176 & 179.88 & 27.13 & 105 & 275 & \multirow{2}{*}{1,298} & \multirow{2}{*}{30.00} & \multirow{2}{*}{.000} \\
\hline & MA & 124 & 199.92 & 36.22 & 130 & 287 & & & \\
\hline \multirow{2}{*}{$\begin{array}{l}\text { Obstacle course } \\
\text { backwards }\end{array}$} & EA & 173 & 112.83 & 27.70 & 58 & 238 & \multirow{2}{*}{1,295} & \multirow{2}{*}{22.70} & \multirow{2}{*}{.000} \\
\hline & MA & 124 & 98.21 & 23.60 & 62 & 160 & & & \\
\hline \multirow{2}{*}{ Sit-ups } & EA & 176 & 47.52 & 9.69 & 20 & 75 & \multirow{2}{*}{1,297} & \multirow{2}{*}{52.91} & \multirow{2}{*}{.000} \\
\hline & MA & 123 & 56.38 & 11.27 & 24 & 85 & & & \\
\hline \multirow{2}{*}{$\begin{array}{l}\text { Forward bend on the } \\
\text { bench }\end{array}$} & EA & 178 & 46.53 & 8.61 & 17 & 66 & \multirow{2}{*}{1,301} & \multirow{2}{*}{21.34} & \multirow{2}{*}{.000} \\
\hline & MA & 125 & 50.86 & 7.15 & 27 & 66 & & & \\
\hline \multirow{2}{*}{ Bent-arm hang } & EA & 173 & 41.11 & 26.72 & 0 & 120 & \multirow{2}{*}{1,295} & \multirow{2}{*}{8.44} & \multirow{2}{*}{.004} \\
\hline & MA & 124 & 49.60 & 21.98 & 0 & 140 & & & \\
\hline \multirow{2}{*}{60 -metre run** } & EA & 177 & 99.69 & 11.34 & 79 & 164 & \multirow{2}{*}{1,266} & \multirow{2}{*}{17.57} & \multirow{2}{*}{.000} \\
\hline & MA & 91 & 93.11 & 13.64 & 74 & 135 & & & \\
\hline \multirow{2}{*}{600 -metre run ${ }^{\star *}$} & EA & 171 & 160.07 & 31.71 & 105 & 263 & \multirow{2}{*}{1,260} & \multirow{2}{*}{2.58} & 110 \\
\hline & MA & 91 & 153.23 & 34.86 & 95 & 265 & & & . 170 \\
\hline
\end{tabular}

Note. $E A$ = early adolescence, $M A=$ middle adolescence; upper-arm skinfold (mm), hand tapping (number of repetitions in 20 seconds) standing long-jump ( $\mathrm{cm}$ ), obstacle course backwards (s/10), sit-ups (number of repetitions in 60 seconds), forward bend on the bench $(\mathrm{cm})$, bent-arm hang (s), 60-metre run (s/10), 600-metre run (s). ANOVA results are for the comparison between the two age groups. * The upper-arm skinfold is a measure of body fat, so the lower value expresses the preferred result. * ${ }^{*}$ For 60 -metre and 600 -metre runs, lower values express better test results. 
Table 2. Descriptive statistics and testing differences between the age groups-self-perceived physical fitness variables (assessed with $P S D Q$ )

\begin{tabular}{|c|c|c|c|c|c|c|c|c|c|}
\hline Variable & $\begin{array}{l}\text { Age } \\
\text { group }\end{array}$ & $\mathrm{N}$ & M & SD & Min & Max & df & $\mathrm{F}$ & Sig. \\
\hline \multirow{2}{*}{ Health } & EA & 162 & 32.54 & 7.18 & 15 & 47 & \multirow{2}{*}{1,298} & \multirow{2}{*}{1.71} & \multirow{2}{*}{.192} \\
\hline & MA & 138 & 33.64 & 7.38 & 8 & 48 & & & \\
\hline \multirow{2}{*}{ Coordination } & EA & 162 & 22.28 & 5.61 & 9 & 36 & \multirow{2}{*}{1,299} & \multirow{2}{*}{.762} & \multirow{2}{*}{.383} \\
\hline & MA & 139 & 21.72 & 5.57 & 9 & 36 & & & \\
\hline \multirow{2}{*}{ Physical activity } & EA & 162 & 24.89 & 6.55 & 6 & 36 & \multirow{2}{*}{1,299} & \multirow{2}{*}{2.037} & \multirow{2}{*}{.155} \\
\hline & MA & 139 & 23.70 & 7.84 & 6 & 36 & & & \\
\hline \multirow{2}{*}{ Body fat } & EA & 162 & 24.83 & 7.62 & 6 & 36 & \multirow{2}{*}{1,299} & \multirow{2}{*}{2.878} & \multirow{2}{*}{.091} \\
\hline & MA & 139 & 26.33 & 7.72 & 6 & 36 & & & \\
\hline \multirow{2}{*}{ Sports competence } & EA & 162 & 22.15 & 6.33 & 6 & 36 & \multirow{2}{*}{1,299} & \multirow{2}{*}{2.728} & \multirow{2}{*}{.100} \\
\hline & MA & 139 & 20.92 & 6.61 & 6 & 36 & & & \\
\hline \multirow{2}{*}{ Appearance } & EA & 162 & 22.31 & 5.62 & 6 & 35 & \multirow{2}{*}{1,299} & \multirow{2}{*}{1,258} & \multirow{2}{*}{.263} \\
\hline & MA & 139 & 23.05 & 5.73 & 7 & 36 & & & \\
\hline \multirow{2}{*}{ Strength } & EA & 162 & 22.53 & 5.27 & 11 & 36 & \multirow{2}{*}{1,299} & \multirow{2}{*}{4.738} & \multirow{2}{*}{.030} \\
\hline & MA & 139 & 21.08 & 6.30 & 8 & 36 & & & \\
\hline \multirow{2}{*}{ Flexibility } & EA & 162 & 23.74 & 5.80 & 7 & 36 & \multirow{2}{*}{1,299} & \multirow{2}{*}{4.474} & \multirow{2}{*}{.035} \\
\hline & MA & 139 & 22.33 & 5.72 & 8 & 36 & & & \\
\hline \multirow{2}{*}{ Fitness/endurance } & EA & 162 & 21.66 & 6.69 & 6 & 36 & 1299 & 2480 & 116 \\
\hline & MA & 139 & 20.40 & 7.15 & 6 & 36 & , & 2. & . \\
\hline Glabal nhysical-self & EA & 162 & 23.72 & 6.81 & 6 & 36 & 1299 & 438 & 509 \\
\hline 10 & MA & 139 & 23.21 & 6.42 & 6 & 36 & 1,200 & . & . \\
\hline Global self-esteem & EA & 162 & 32.97 & 7.10 & 10 & 48 & 1299 & 3749 & 054 \\
\hline . & MA & 139 & 34.54 & 6.85 & 10 & 48 & (3) & & \\
\hline
\end{tabular}

Note. $E A=$ early adolescence, $M A=$ middle adolescence. ANOVA results are for the comparison between the two age groups.

Table 3. Adjusted mean values and ANCOVA for testing differences between the age groups adjusted for the covariate (gender) actual physical fitness variables (assessed with Sports-education Chart)

\begin{tabular}{|c|c|c|c|c|c|c|}
\hline Variable & Age group & df & M & $\mathrm{F}$ & $\mathrm{p}$ & $\eta 2$ \\
\hline \multirow{2}{*}{ Upper-arm skinfold } & EA & \multirow[t]{2}{*}{1,302} & 12.290 & \multirow[t]{2}{*}{.260} & \multirow[t]{2}{*}{.611} & \multirow[t]{2}{*}{.001} \\
\hline & MA & & 12.622 & & & \\
\hline \multirow{2}{*}{ Hand tapping } & EA & \multirow{2}{*}{1,301} & 42.386 & \multirow[t]{2}{*}{114.978} & \multirow[t]{2}{*}{.000} & \multirow[t]{2}{*}{.276} \\
\hline & MA & & 48.367 & & & \\
\hline \multirow{2}{*}{ Standing long jump } & EA & \multirow[t]{2}{*}{ 1, 297} & 178.531 & \multirow[t]{2}{*}{57.996} & \multirow[t]{2}{*}{.000} & \multirow[t]{2}{*}{.163} \\
\hline & MA & & 201.835 & & & \\
\hline \multirow{2}{*}{$\begin{array}{l}\text { Obstacle course } \\
\text { backwards }\end{array}$} & EA & \multirow[t]{2}{*}{ 1, 294} & 113.572 & \multirow[t]{2}{*}{32.211} & \multirow[t]{2}{*}{.000} & \multirow[t]{2}{*}{.099} \\
\hline & MA & & 97.169 & & & \\
\hline \multirow{2}{*}{ Sit-ups } & EA & \multirow[t]{2}{*}{1,296} & 47.355 & \multirow[t]{2}{*}{59.680} & \multirow[t]{2}{*}{.000} & \multirow[t]{2}{*}{.168} \\
\hline & MA & & 56.614 & & & \\
\hline \multirow{2}{*}{$\begin{array}{l}\text { Forward bend on the } \\
\text { bench }\end{array}$} & EA & \multirow[t]{2}{*}{1,300} & 46.750 & \multirow[t]{2}{*}{18.335} & \multirow[t]{2}{*}{.000} & \multirow[t]{2}{*}{.058} \\
\hline & MA & & 50.548 & & & \\
\hline \multirow{2}{*}{ Bent-arm hang } & EA & \multirow[t]{2}{*}{1,294} & 40.585 & \multirow[t]{2}{*}{11.783} & \multirow[t]{2}{*}{.001} & \multirow[t]{2}{*}{.039} \\
\hline & MA & & 50.338 & & & \\
\hline \multirow{2}{*}{ 60-metre run } & EA & 1,265 & 99.908 & 23.186 & .000 & .080 \\
\hline & MA & & 92.878 & & & \\
\hline $0 \cap 0$ motro nu & EA & 1,259 & 160.585 & 4.919 & .027 & .019 \\
\hline bov-metre run & MA & & 152.264 & & & \\
\hline
\end{tabular}

Note. Covariate $=$ variable: gender,$E A=$ early adolescence,$M A=$ middle adolescence, $\mathrm{df}=$ degrees of freedom; $\mathrm{M}=$ adjusted mean, $F=$ Fisher's statistics, $p=$ level of statistical significance, $\eta^{2}=$ partial eta squared. 
gender as a covariate. There was a significant effect $(p<.05)$ of age on the dependent variables of hand tapping, standing long jump, obstacle course backward, sit-ups, forward bending on the bench, bentarm hang, 60- and 600-metre run after controlling for gender. There was no significant effect ( $p>05)$ of age on upper arm skinfold after controlling for gender. The partial eta squared values, varying from .019 to .276 (for the variables with a significant effect of age), indicated that the magnitude of their effect was small (Cohen, 1988).

Table 4 shows the adjusted means and ANCOVA used to test the differences between the two age groups for the self-perceived physical fitness variables measured by PSDQ, adjusted for gender as a covariate. Results showed a significant effect $(p<.05)$ of age on the dependent variables of body fat, strength, flexibility, and global self-esteem after controlling for gender. There was no significant effect ( $p>.05)$ of age on health, coordination, physical activity, sports competence, appearance, fitness/endurance, and global physical self-esteem after controlling for gender. The effect of all variables was very weak (the partial eta squared values varied between .001 and .014).

\section{Correlation between the actual and self- perceived physical fitness}

Table 5 shows the correlation coefficients between the variables SEC and PSDQ for the entire sample of participants. The comparison between SEC and PSDQ showed several significant correlations. Self-perceived physical activity, appearance, flexibility, and general self-esteem correlated strongly with seven out of nine actual physical fitness measures (see Table 5). The percentage of body fat (as measured by the upper arm skinfold), coordination of the whole-body movements (i.e., obstacle course backward), explosive strength (i.e., standing long jump), sprint speed (i.e., 60-metre run), and general endurance (i.e., 600-metre run) correlated strongly with almost all the self-perceived measures of physical self (significant correlations with nine or 10 out of 11 measures). The hand tapping test, which measures the speed of alternating movements, correlated with only one dimension of physical self, namely perception of health.

Tables 6 and 7 show correlations between various measures of actual and self-perceived physical fitness for the two age groups-Table 6 for the early adolescent group and Table 7 for the

Table 4. Adjusted mean values and ANCOVA for testing differences between the age groups adjusted for the covariate (gender)self-perceived physical fitness variables (assessed with PSDQ)

\begin{tabular}{|c|c|c|c|c|c|c|}
\hline Variable & Age group & $d f$ & M & $\mathrm{F}$ & $\mathrm{p}$ & $\eta 2$ \\
\hline \multirow{2}{*}{ Health } & EA & 1,297 & 32.491 & \multirow{2}{*}{2.148} & \multirow{2}{*}{.144} & \multirow{2}{*}{.007} \\
\hline & MA & & 33.706 & & & \\
\hline \multirow{2}{*}{ Coordination } & EA & 1,298 & 22.237 & \multirow{2}{*}{.545} & \multirow{2}{*}{.461} & \multirow{2}{*}{.002} \\
\hline & MA & & 21.774 & & & \\
\hline \multirow{2}{*}{ Physical activity } & EA & 1,298 & 24.840 & \multirow{2}{*}{1.753} & \multirow{2}{*}{.187} & \multirow{2}{*}{.006} \\
\hline & MA & & 23.762 & & & \\
\hline \multirow{2}{*}{ Body fat } & EA & 1,298 & 24.743 & \multirow{2}{*}{4.020} & \multirow{2}{*}{.046} & \multirow{2}{*}{.013} \\
\hline & MA & & 26.429 & & & \\
\hline \multirow{2}{*}{ Sports competence } & EA & 1,298 & 22.102 & \multirow{2}{*}{2.377} & \multirow{2}{*}{.124} & \multirow{2}{*}{.008} \\
\hline & MA & & 20.982 & & & \\
\hline \multirow{2}{*}{ Appearance } & EA & 1,298 & 22.275 & \multirow{2}{*}{1.630} & \multirow{2}{*}{.203} & \multirow{2}{*}{.005} \\
\hline & MA & & 23.096 & & & \\
\hline \multirow{2}{*}{ Strength } & EA & 1,298 & 22.475 & \multirow{2}{*}{4.319} & \multirow{2}{*}{.039} & \multirow{2}{*}{.014} \\
\hline & MA & & 21.145 & & & \\
\hline \multirow{2}{*}{ Flexibility } & EA & 1,298 & 23.696 & \multirow{2}{*}{4.077} & \multirow{2}{*}{.044} & \multirow{2}{*}{.013} \\
\hline & MA & & 22.383 & & & \\
\hline \multirow{2}{*}{ Fitness/ endurance } & EA & 1, 298 & 21.595 & \multirow{2}{*}{2.109} & & \\
\hline & MA & & 20.480 & & .148 & .007 \\
\hline Clohbloburiol olf & EA & 1,298 & 23.670 & 000 & 500 & \\
\hline Giondi pilysical-sen & MA & & 23.263 & .290 & .509 & .001 \\
\hline Clopol colf octoom & EA & 1,298 & 32.940 & 1102 & 011 & 014 \\
\hline Godal sen-esteemi & MA & & 34.580 & 4.192 & .041 & .014 \\
\hline
\end{tabular}

Note. Covariate = variable: gender, $\mathrm{EA}=$ early adolescence, $\mathrm{MA}=$ middle adolescence df $=$ degrees of freedom, $\mathrm{M}=$ adjusted mean, $\mathrm{F}=$ Fisher's statistics, $\mathrm{p}=$ level of statistical significance, $\eta^{2}=$ partial eta squared. 
middle adolescent group. Compared to the correlations for the total sample of participants, there were fewer significant correlations between the measured variables in each age group. In early adolescence, self-perceived physical activity correlates strongly with body fat percentage (upper arm skinfold) and flexibility (forward bend on the bench), while correlations with the total body coordination (obstacle course backward), abdominal muscle strength (sit-ups), and general endurance (600-metre run test) were moderate. Self-perceived strength did not correlate with any of the actual physical fitness measures, and self-perceived health correlated moderately with only one variable - body fat percentage (upper arm skinfold).
Table 7 shows correlations for the older age group. Several self-perceived measures of physical self (physical activity, body fat, sports competence, flexibility, and fitness/endurance) correlated moderately to strongly with half of the actual measures of physical fitness (six out of 12). Self-perceived health did not correlate with any of the physical fitness measures. In mid-adolescence, explosive strength (standing long jump) and sprint speed (60-metre run) correlated strongly with the majority of the measures associated with self-perception (10 and nine out of 10 , respectively).

Table 5. Pearson correlation coefficients between the Sports-educational chart and PSDQ — all participants

\begin{tabular}{|c|c|c|c|c|c|c|c|c|c|c|c|}
\hline $\begin{array}{l}\text { Actual hysical } \\
\text { fitness }\end{array}$ & $\mathrm{H}$ & C & PA & $\mathrm{BF}$ & SC & GPS & A & $S$ & $\mathrm{~F}$ & F/E & GSE \\
\hline $\begin{array}{l}\text { Upper-arm } \\
\text { skinfold }\end{array}$ & -.088 & $-.303^{* *}$ & $-.274^{* *}$ & $-.460^{* *}$ & $-.334^{* *}$ & $-.300^{* *}$ & $-.212^{* *}$ & $-.17^{* *}$ & $-.348^{* *}$ & $-.332^{* *}$ & $-.277^{* *}$ \\
\hline Hand tapping & $.151^{*}$ & -.048 & -.015 & .088 & .000 & .034 & .014 & -.068 & -.038 & -.049 & .079 \\
\hline $\begin{array}{l}\text { Standing long- } \\
\text { jump }\end{array}$ & .124 & $.188^{* *}$ & $.255^{* *}$ & $.180^{* *}$ & $.281^{* *}$ & $.178^{* *}$ & $.185^{*}$ & .123 & $.213^{* *}$ & $.167^{*}$ & $.271^{* *}$ \\
\hline $\begin{array}{l}\text { Obstacle } \\
\text { course } \\
\text { backwards }\end{array}$ & $-.167^{*}$ & $-.149^{*}$ & $-.135^{*}$ & $-.261^{* *}$ & $-.190^{* *}$ & $-.140^{*}$ & $-.183^{* *}$ & .011 & $-.192^{* *}$ & $-.167^{*}$ & $-.190^{* *}$ \\
\hline Sit-ups & -.069 & $.137^{*}$ & $.196^{* *}$ & .057 & $.251^{* *}$ & .105 & $.144^{*}$ & .060 & $.153^{*}$ & $.165^{*}$ & $.143^{*}$ \\
\hline $\begin{array}{l}\text { Forward bend } \\
\text { on the bench }\end{array}$ & $.161^{*}$ & .050 & $.183^{* *}$ & -.007 & .056 & .021 & $-.138^{*}$ & -.009 & $.189^{* *}$ & .095 & .073 \\
\hline Bent-arm hang & .050 & .007 & -.055 & $.272^{* *}$ & -.004 & .070 & .087 & $.154^{*}$ & .029 & .025 & $.146^{*}$ \\
\hline 60-metre run & $-.152^{*}$ & $-.185^{* *}$ & $-.193^{* *}$ & -.124 & $-.285^{* *}$ & $-.217^{* *}$ & $-.212^{* *}$ & -.112 & $-.208^{* *}$ & $-.192^{* *}$ & $-.331^{* *}$ \\
\hline 600-metre run & -.048 & $-.229^{* *}$ & $-.245^{* *}$ & $-.200^{* *}$ & $-.318^{* *}$ & $-.211^{* *}$ & $-.176^{*}$ & -.014 & $-.224^{* *}$ & $-.349^{* *}$ & $-.195^{* *}$ \\
\hline
\end{tabular}

Note. $\mathrm{H}=$ health, $\mathrm{C}=$ coordination, $\mathrm{PA}=$ physical activity, $\mathrm{BF}=$ body fat, $\mathrm{SC}=$ sports competence, $\mathrm{GPS}=$ global physical-self, $\mathrm{A}$ $=$ appearance, $\mathrm{S}=$ strength, $\mathrm{F}=$ flexibility, $\mathrm{F} / \mathrm{E}=$ fitness $/$ endurance, $\mathrm{GSE}=$ global self-esteem. Significant Pearson correlations: * $p<.05,{ }^{* *} p<.001$.

Table 6. Pearson correlation coefficients between the Sports-educational chart and PSDQ-early adolescence

\begin{tabular}{|c|c|c|c|c|c|c|c|c|c|c|c|}
\hline $\begin{array}{l}\text { Actual physical } \\
\text { fitness }\end{array}$ & $\mathrm{H}$ & C & PA & $\mathrm{BF}$ & SC & GPS & A & $S$ & $\mathrm{~F}$ & $\mathrm{~F} / \mathrm{E}$ & GSE \\
\hline $\begin{array}{l}\text { Upper-arm } \\
\text { skinfold }\end{array}$ & $-.165^{*}$ & $-.257^{* *}$ & $-.231^{* *}$ & $-.478^{* *}$ & $-.296^{* *}$ & $-.305^{* *}$ & $-.255^{* *}$ & -.134 & $-.281^{* *}$ & $-.283^{* *}$ & $-.285^{* *}$ \\
\hline Hand tapping & .096 & -.122 & .005 & .020 & -.049 & .008 & -.084 & -.156 & -.048 & -.055 & -.051 \\
\hline $\begin{array}{l}\text { Standing long- } \\
\text { jump }\end{array}$ & .094 & .038 & .115 & .025 & .122 & .091 & .089 & .020 & .117 & .002 & .141 \\
\hline $\begin{array}{l}\text { Obstacle } \\
\text { course } \\
\text { backwards }\end{array}$ & -.142 & -.130 & $-.186^{*}$ & $-.225^{* *}$ & -.164 & -.119 & -.149 & .050 & $-.202^{*}$ & -.162 & -.107 \\
\hline Sit-ups & -.139 & .084 & $.170^{*}$ & -.137 & $.243^{* *}$ & .094 & .048 & -.002 & .121 & .162 & .080 \\
\hline $\begin{array}{l}\text { Forward bend } \\
\text { on the bench }\end{array}$ & .158 & .047 & $.236^{* *}$ & .053 & .088 & .031 & -.110 & -.042 & $.237^{* *}$ & .089 & .102 \\
\hline Bent-arm hang & .111 & .029 & .109 & $.181^{*}$ & .104 & $.184^{*}$ & .061 & .060 & .116 & .144 & .120 \\
\hline 60-metre run & -.095 & -.102 & -.102 & .008 & $-.215^{*}$ & $-.185^{*}$ & $-.171^{*}$ & -.064 & -.139 & -.110 & $-.217^{* *}$ \\
\hline 600 -metre run & -.056 & $-.184^{*}$ & $-.187^{\star}$ & $-.201^{*}$ & $-.285^{* *}$ & $-.204^{*}$ & $-.185^{*}$ & -.078 & $-.170^{*}$ & $-.260^{* *}$ & -.141 \\
\hline
\end{tabular}

Note. $\mathrm{H}=$ health, $\mathrm{C}=$ coordination, $\mathrm{PA}=$ physical activity, $\mathrm{BF}=$ body fat, $\mathrm{SC}=$ sports competence, $\mathrm{GPS}=$ global physical-self, $\mathrm{A}$ = appearance, $\mathrm{S}=$ strength, $\mathrm{F}=$ flexibility, $\mathrm{F} / \mathrm{E}=$ fitness/ endurance, GSE = global self-esteem. Significant Pearson correlations: * $\mathrm{p}<.05,{ }^{* *} \mathrm{p}<.001$. 
Table 7. Pearson correlation coefficients between the Sports-educational chart and PSDQ-middle adolescence

\begin{tabular}{|c|c|c|c|c|c|c|c|c|c|c|c|}
\hline $\begin{array}{l}\text { Actual hysical } \\
\text { fitness }\end{array}$ & $\mathrm{H}$ & C & PA & $\mathrm{BF}$ & SC & GPS & A & $S$ & $\mathrm{~F}$ & $\mathrm{~F} / \mathrm{E}$ & GSE \\
\hline $\begin{array}{l}\text { Upper-arm } \\
\text { skinfold }\end{array}$ & -.017 & $-.354^{* *}$ & $-.311^{* *}$ & $-.495^{* *}$ & $-.374^{* *}$ & $-.314^{* *}$ & -.195 & -.201 & $-.417^{* *}$ & $-.383^{* *}$ & $-.346^{* *}$ \\
\hline Hand tapping & .147 & .092 & -.001 & .072 & .143 & .069 & .054 & .159 & .092 & .025 & .095 \\
\hline $\begin{array}{l}\text { Standing long- } \\
\text { jump }\end{array}$ & .127 & $.429^{* *}$ & $.460^{* *}$ & $.359^{* *}$ & $.562^{* *}$ & $.330^{* *}$ & $.292^{* *}$ & $.319^{* *}$ & $.430^{* *}$ & $.447^{* *}$ & $.423^{* *}$ \\
\hline $\begin{array}{l}\text { Obstacle } \\
\text { course } \\
\text { backwards }\end{array}$ & -.166 & -.203 & -.104 & $-.272^{* *}$ & $-.276^{* *}$ & -.181 & -.203 & -.097 & $-.249^{*}$ & $-.227^{\star}$ & $-.261^{*}$ \\
\hline Sit-ups & -.028 & $.246^{*}$ & $.270^{* *}$ & $.263^{*}$ & $.322 *$ & .120 & $.233^{*}$ & .204 & $.280^{* *}$ & $.235^{*}$ & .139 \\
\hline $\begin{array}{l}\text { Forward bend } \\
\text { on the bench }\end{array}$ & .121 & .075 & .138 & -.189 & .032 & -.004 & $-.241^{*}$ & .092 & .170 & .145 & -.082 \\
\hline Bent-arm hang & -.066 & -.014 & $-.218^{*}$ & $.382^{* *}$ & -.130 & -.094 & .107 & $.273^{* *}$ & -.063 & -.107 & .166 \\
\hline 60-metre run & -.238 & $-.406^{* *}$ & $-.353^{* *}$ & $-.342^{* *}$ & $-.492^{* *}$ & $-.306^{*}$ & $-.266^{*}$ & -.228 & $-.431^{* *}$ & $-.381^{* *}$ & $-.564^{* *}$ \\
\hline 600-metre run & -.026 & $-.315^{*}$ & $-.328^{*}$ & -.203 & $-.390^{* *}$ & -.231 & -.161 & .075 & $-.328^{*}$ & $-.498^{* *}$ & $-.341^{* *}$ \\
\hline
\end{tabular}

Note. $\mathrm{H}=$ health, $\mathrm{C}=$ coordination, $\mathrm{PA}=$ physical activity, $\mathrm{BF}=$ body fat, $\mathrm{SC}=$ sports competence, GPS = global physical-self, $\mathrm{A}$ $=$ appearance, $\mathrm{S}=$ strength, $\mathrm{F}=$ flexibility, $\mathrm{F} / \mathrm{E}=$ fitness/ endurance, GSE = global self-esteem. Significant Pearson correlations: * $p<.05,{ }^{* *} p<.001$.

\section{Discussion}

The purpose of this study was twofold: first, to analyse age-related differences in actual and selfperceived physical fitness measures, and second, to evaluate the relationship between self-perceived and objective measures of physical fitness.

In terms of age, the older group of adolescents scored better on all but two measured physical fitness tests. These results confirm the development of alternating movement speed, explosive strength, the whole body coordination, abdominal strength, flexibility, muscular endurance of the shoulder girdle, and sprinting speed over the course of early to mid-adolescence. There were no differences in body fat percentage (upper arm skinfold) and general endurance (600-metre run). Although the lack of increase in body fat percentage is positive as it shows that adolescents generally do not gain body fat over the course of three years, the lack of development in general endurance is alarming. These results imply that the level of general endurance remains the same between early and mid-adolescence over the course of three years. Since cardiorespiratory and muscular fitness have been shown to be associated with the established and emerging risk factors for cardiovascular disease (Ortega, Ruiz, Castillo, \& Sjostrom, 2008), promoting activities that improve the level of general endurance in adolescents is very important. The results of our study show that the age of the subjects explains the differences in physical fitness with statistical significance, which is consistent with the findings of numerous other authors (Huotari, Sääkslahti, \& Watt, 2009; Malina, et al., 2004; Matejek \& Starc, 2013; Starc \& Strel, 2010; Starc, Strel, \& Kovač, 2010; Strel, et al., 2003).
Age-related differences in self-perceived physical fitness were evident in only two of the specific measures of physical self, i.e., perceptions of strength and flexibility, and in one global measure - global self-esteem. The results suggest that the older adolescents had poorer self-perceptions of strength and flexibility, while on the other hand, they were more self-aware of who they were and how they were perceived by their social environment. The results of this cross-sectional study replicate those by Marsh (1993), who documented almost no changes in physical self-perceptions during adolescence. Most studies using multidimensional instruments report that general physical perception increases later in adolescence and early adulthood (Sonstroem, 1998). Several authors (for a review see Marsh, 1993) describe a general decline in physical self-esteem in adolescence, with a significant drop between the ages of 12 and 16 (Sonstroem, 1998).

Correlations were determined between the physical fitness test scores and PSDQ scales, which are measures of physical self-concept. The results showed many significant correlations between the specific motor skills and the physical self scales, especially when the entire sample was observed. Interestingly, the calculations that included only the younger or only the older group of participants showed fewer significant correlations. Based on these results, we can assume that the relationship between general physical self-concept and psychosomatic status is not direct and general, but rather isolated and specific, i.e. correlated with different aspects of physical fitness. These assumptions were confirmed by Jaakkola and colleagues (2019), who reported the reciprocal nature of the relationships 
between motor skill development, perceived physical competence, health-related fitness, and objectively measured physical activity.

The results showed that specific motor skills, as measured by physical fitness tests, were significantly correlated with both the general self-concept and various aspects of physical self-concept. As in the study by Dunton, Schneider, Graham, and Cooper (2006), the results confirmed that endurance appeared to be positively related to perceptions of being physically active on a regular basis, being good at coordinated movements, being good at sports and having good athletic skills, having less body fat, having a more favourable appearance, and being more flexible and fit. Endurance is positively related to global self-esteem and feeling positive about oneself physically. Results also show that the percentage of body fat, as measured by the upper arm skinfold, correlates significantly with all dimensions of physical self except perception of health. Global self-esteem correlates with body fat percentage, explosive strength, coordination, abdominal muscle strength, muscular endurance of the shoulder girdle and arms, sprint speed, and overall endurance. Global physical self correlates with the same motor dimensions, except for abdominal muscle strength and shoulder girdle muscular endurance.

Correlation analysis shows that in early adolescence fewer than one-third of the correlations (29 out of 99) are significant, whereas in middle adolescence half of the correlations are significant (49 out of 99). Calculations for the entire sample show two-thirds significant correlations (62 of 99). These findings are congruent with those of Marsh and Redmayne (1994), who report relatively weak and few correlations between actual physical fitness and various aspects of the physical self. (Rahmani-Nia, Damitchi, Azizi, and Hoseini, (2011) interpret a rather weak and non-significant association between perceived body composition and skinfold thickness as a result of inaccurate self-assessments by adolescents. It is likely that younger adolescents' self-assessments are strongly influenced by peerrelated norms, which may lead to a relative discrepancy between the actual and perceived physical fitness. As adolescence is a developmental period associated with a number of significant biological, social and psychological changes, these may also influence health-related quality of life (Cumming, et al., 2011).

Further, this implies that physical fitness is not as closely related to the development of the physical self as had been assumed. Borrego Balsalobrea, López Sáncheza, and Díaz Suárez (2014) report the relationship between endurance and strength as components of physical fitness and the factors that determine physical self-concept. It can be assumed that kinaesthetic sensations only partially contribute to the development of physical selfconcept (Sonstroem, 1998). Therefore, weak correlations are not surprising, but it must be emphasised that motor skills have a stronger relationship with the physical self when viewed in a social context, through feedback information, or in comparison with others. In this way, pure kinaesthetic sensation is complemented by subjective interpretation and social comparison, leading to a more or less positive physical self-concept.

The overall view of the results shows that physical self-concept and actual indicators of physical fitness are related, despite incomplete overlap between psychological dimensions and objective measurements. According to Sonstroem (1998), kinaesthetic feelings only partially contribute to the development of physical self-concept. It should be emphasised that motor skills have a stronger relation to the physical self when viewed in a social context, through feedback information, or in comparison with others. In this way, pure kinaesthetic sensations are complemented by subjective interpretation and social comparison, leading to a more or less positive physical self-concept.

The obtained results can be transferred to physical education, where the development of a positive physical self-concept is one of the most important educational goals. Although physical education is an important environment in which the development and formation of physical self-concept takes place, other aspects such as the influence of significant others must also be taken into account (Jurak, Kovač, \& Strel, 2006). These results show us that specific motor skills are closely related to their subjective representations, i.e. aspects of the physical self. 


\section{References}

Bezjak, R. (2016). Vpliv gibalnih sposobnosti in izbranih psiholoških vidikov športne vzgoje na telesno samopodobo učencev. [Impact of physical abilities and selected psychological aspects of physical education on students' physical self-concept. In Slovenian.] (Doctoral dissertation, University of Ljubljana, Faculty of Sport) University of Ljubljana Digital Archive. https:/www.fsp.uni-lj.si/COBISS/Dr/Doktorat22M00143BezjakRobert.pdf 201505010014301160751581

Borrego Balsalobrea, F.J., López Sáncheza, G.F., \& Díaz Suáreza, A. (2014). Relationships between physical fitness and physical self-concept in Spanish adolescents. Procedia - Social and Behavioral Sciences, 132, 343-350.

https://doi.org/10.1016/j.sbspro.2012.11.458

Carcamo-Oyarzun, J., Estevan, I., \& Herrmann, C. (2020). Association between actual and perceived motor competence in school children. International Journal of Environmental Research and Public Health, 17, 3408.

https://doi.org/10.3390/ijerph17103408

Carraro, A., Scarpa, S., \& Ventura, L. (2010). Relationships between physical self-concept and physical fitness in Italian adolescents. Perceptual and Motor Skills, 110(2), 522-530. https://doi.org/10.2466/pms.110.2.522-530 201505012336561148134351

Cohen, J. (1988). Statistical power analysis for the behavioral sciences. Erlbaum: Hillsdale.

Cumming, S.P., Standage, M., Loney, T., Gammon, C., Neville, H., Sherar, L.B., \& Malina, R.M. (2011). The mediating role of physical self-concept on relations between biological maturity status and physical activity in adolescent females. Journal of Adolescence, 34, 465-473. https://doi.org/10.1016/j.adolescence.2010.06.006 20150502110311719353556

Dunton, G.F., Schneider, M., Graham, D.J., \& Cooper, D.M. (2006). Physical activity, fitness, and physical selfconcept in adolescent females. Pediatric Exercise Science, 18, 240-251. https://doi.org/1010.1123/pes.18.2.240 20150502163906268584013

Fox, K.R. (1998). Advances in the measurement of physical self. In J.L. Duda (Ed.), Advances in sport and exercise psychologymeasurement (pp. 295-310). Morgantown: Fitness Information Technology. 20150503023129940586090

Hagger, M.S., Chatzisarantis, N.L.D., Biddle, S.J.H., \& Orbell, S. (2001). Antecedents of children's physical activity intentions and behaviour: Predictive validity and longitudinal effects. Psychology and Health, 16, 391-407. https://doi.org/10.1080/08870440108405515 201505030354501865887046

Huotari, P., Sääkslahti, A., \& Watt, A. (2009). Associations between the self-estimated and actual physical fitness scores of Finnish grade 6 students. Facta Universitatis, 7(1), 27-36. 20150501233853453264236

Jaakkola, T., Huhtiniemi, M., Salin, K., Seppälä, S., Lahti, J., Hakonen, H., \& Stodden, D.F. (2019). Motor competence, perceived physical competence, physical fitness, and physical activity within Finnish children. Scandinavian Journal of Medicine and Science in Sports, 29, 7, 1013-1021. https://doi.org/1010.1111/sms.13412

Jaakkola, T., \& Washington, T.L. (2011). Measured and perceived physical fitness, intention and self-reported physical activity in adolescence. Advances in Physical Education, 1(2), 16-22. https://doi.org/10.4236/ape.2011.12004 20150501234250459153414

Jurak, G., Kovač, M., \& Strel, J. (2006). Impact of the additional physical education lessons programme of the physical and motor development of 7- to 10-year-old children. Kinesiology, 38(2), 105-115. 201505012341181969484687

Kovač, M., Jurak, G., Starc, G., Leskošek, B., \& Strel, J. (2011). Športnovzgojni karton: Diagnostika in ovrednotenje telesnega in gibalnega razvoj otrok in mladine v Sloveniji. [Sports-educational Chart: Diagnostics and evaluation of physical and motor development of children and youth in Slovenia. In Slovenian.] Ljubljana: Fakulteta za šport.

Labbrozzi, D., Robazza, C., Bertollo, M., Bucci, I., \& Bortoli, L. (2013). Pubertal development, physical self-perception, and motivation toward physical activity in girls. Journal of Adolescence, 36, 759-765. https://doi.org/1010.1016/j. adolescence.2013.06.002 20150501234813991739750

Lintunen, T. (1999). Development of self-perceptions during the school years. In Y. Vanden Auweele, F. Bakker, S. Biddle, M. Durand \& R. Seiler (Eds.), Psychology for physical educators (pp. 115-134). Champaign: Human Kinetics. 201504302147471673638344

Malina, R.M., Bouchard, C., \& Bar-Or, O. (2004). Growth, maturation, and physical activity. Champaign: Human Kinetics. 20150502113550653483391

Marsh, H.W. (1993). Physical fitness self-concept: Relations of physical fitness to field and technical indicators for boys and girls aged 9-15. Journal of Sport and Exercise Psychology, 15, 184-206. 20150502135126276080608

Marsh, H.W., \& Redmayne, R.S. (1994). A multidimensional physical self-concept and its relation to multiple components of physical fitness. Journal of Sport and Exercise Psychology, 16, 43-55. 201505030206561742427946

Marsh, H.W., Richards, G.E., Johnson, S., Roche, L., \& Tremayne, P. (1994). Physical Self-Description Questionnaire: Psychometric properties and multitrait-multimethod analysis of relations to existing instruments. Journal of Sport and Exercise Psychology, 16, 270-305. 201505010006321246777892

Matejek, Č., \& Starc, G. (2013). The relationship between children's physical fitness and gender, age and environmental factors. Annales Kinesiologiae, 4(2), 17-30. 20150502113902238211989 
McIntyre, F., Parker, H., Chivers, P., \& Hands, B. (2018). Actual competence, rather than perceived competence, is a better predictor of physical activity in children aged 6-9 years. Journal of Sports Sciences, 36 (13), 1433-1440. https://doi.org/1010.1080/02640414.2017.1390243

Ortega, F.B., Ruiz, J.R., Castillo, M.J., \& Sjostrom, M. (2008). Physical fitness in childhood and adolescence: A powerful marker of health. Journal of Obesity, 32, 1-11. https://doi.org/10.1038/sj.ijo.0803774 20150502130328671465158

Rahmani-Nia, F., Damitchi, A., Azizi, M., \& Hoseini, R. (2011). Associations between self-perceived and measured physical fitness of male college students. World Applied Sciences Journal, 14(9), 1331-1338. 2015050123445458537125

Sonstroem, R.J. (1998). Physical self-concept: Assessment and external validity. Medicine and Science in Sports and Exercise, 21(3), 329-337. 201505021356001043393970

Starc, G., \& Strel, J. (2010). Tracking excess weight and obesity from childhood to young adulthood: A 12-year prospective cohort study in Slovenia. Public Health Nutrition, 14(1), 49-55. https://doi.org/10.1017/S1368980010000741 20150502113102894452810

Starc, G., Strel, J., \& Kovač, M. (2010). Telesni in gibalni razvoj slovenskih otrok in mladine v številkah: Šolsko leto 2007/2008. [Physical and motor development of Slovenian children and youth in numbers: School year 2007/2008. In Slovenian.] Ljubljana: Faculty of Sport. 201505021134091374282003

Strel, J., Ambrožič, F., Kondrič, M., Kovač, M., Leskošek, B., \& Štihec, J. (1997). Sports-educational chart. Ljubljana: Ministry of Education and Sport. 201505010029071279792428

Strel, J., Kovač, M., Jurak, G., Bednarik, J., Leskošek, B., \& Starc, G. (2003). Nekateri morfološki, gibalni, funkcionalni in zdravstveni parametri otrok in mladine v Sloveniji v letih 1900-2000. [Some morphological, motor, functional and health parameters of children and youth in Slovenia in the years 1900-2000. In Slovenian] Ljubljana: Faculty of Sport. 201505021326391104920983

Submitted: May 7, 2015

Accepted: February 2, 2021

Published Online First: March 31, 2021

Correspondence to:

Saša Cecić Erpič, Ph.D.

University of Ljubljana, Faculty of Sport

Gortanova 22, 1000 Ljubljana, Slovenia

Phone: +38615207735

Fax: +38615207730

E-mail: sasa.cecicerpic@fsp.uni-lj.si 